\title{
Note on the Round-Off Errors in Iterative Processes
}

\section{By J. Descloux}

Summary. This paper discusses round-off errors in iterative processes for solving equations. Let $x_{n+1}=x_{n}+F\left(x_{n}\right)$ be a scalar iterative converging process; the different values $x_{n}$ are represented in a computer with a certain precision; when $x_{n}$ is close to the limit, $F\left(x_{n}\right)$ is small and can perhaps be obtained easily with a higher absolute precision than $x_{n}$; consequently, the addition $x_{n}+F\left(x_{n}\right)$ will practically involve a rounding operation. Besides some general remarks, it will be shown that for a fixed-point computer an appropriate rounding method can provide a more accurate solution to the problem; analogous results are given in Appendix I for a floating-point computer; Appendix II deals with Aitken's $\delta^{2}$ process. The author is indebted to A. H. Taub for many suggestions and stimulating discussions.

1. Introduction. Let $G^{(1)}, \cdots G^{(m)}$ be $m$ real functions of the real variables $x^{(1)}, \cdots x^{(m)}$. For any set of $m$ numbers $p^{(1)}, \cdots p^{(m)}$, we shall use the vectorial notations:

$$
\begin{aligned}
\mathbf{p} & =\left(p^{(1)}, \cdots p^{(m)}\right) ; \\
|\mathbf{p}| & =\sqrt{\left(p^{(1)}\right)^{2}+\cdots\left(p^{(m)}\right)^{2}} .
\end{aligned}
$$

We consider the iterative process

$$
\mathbf{x}_{n+1}=\mathbf{G}\left(\mathbf{x}_{n}\right), \quad n=0,1, \cdots
$$

and suppose there exist a vector $\mathrm{r}$ and a number $b(0 \leqq b<1)$ such that

$$
|\mathbf{G}(\mathbf{x})-\mathbf{r}| \leqq b|\mathbf{x}-\mathbf{r}| \text { for all } \mathbf{x}
$$

the condition (2) insures the convergence of the $\mathbf{x}_{n}$ 's to $\mathbf{r}$.

We want to realize the process (1) on a fixed-point computer under the two conditions: a) For representing each of the $x_{n}{ }^{(i)}$, we use only one "word"; we consider the content of the word as an integer; b) We may use higher precision for computing the values of the functions $G^{(1)}, \cdots G^{(m)}$ (or the functions $G^{(1)}$ $\left.x^{(1)}, G^{(2)}-x^{(2)}, \cdots G^{(m)}-x^{(m)}\right)$.

We distinguish two types of errors:

1) Truncation errors; even when using double precision, we cannot expect to evaluate the functions $G^{(i)}$ exactly;

2) Round-off errors; according to condition a), the value found for $G^{(i)}$ must be rounded to an integer. tion.

Received June 1, 1962. This work was supported in part by the National Science Founda- 
2. Truncation Errors. Let $H^{(1)}(\mathbf{x}), \cdots H^{(m)}(\mathbf{x})$ approximate the functions $G^{(1)}(\mathbf{x}), \cdots G^{(m)}(\mathbf{x})$ :

$$
H^{(i)}(\mathbf{x})=G^{(i)}(\mathbf{x})+\xi^{(i)}(\mathbf{x}) ;
$$

$\xi^{(i)}(\mathbf{x})$ is called the truncation error; it is supposed to satisfy the inequality

$$
\left|\xi^{(i)}(\mathbf{x})\right| \leqq a^{(i)} ; \quad a^{(i)}=\text { constant. }
$$

The iterative process

$$
\mathrm{V}_{n+1}=\mathbf{H}\left(\mathrm{V}_{n}\right)
$$

is considered as an approximation of (1) and gives some information about $r$.

Theorem 1. For any $\mathbf{V}_{0}$, the sequence $\mathrm{V}_{n}$ given by (4) is bounded and all its points of accumulation $\mathbf{V}$ satisfy the inequality

$$
|\mathbf{V}-\mathbf{r}| \leqq \frac{|\mathbf{a}|}{1-b} ; \quad \mathbf{a}=\left(a^{(1)}, \cdots a^{(m)}\right) \text {. }
$$

Theorem 2. The process (4) is. the best possible in the following sense: for given a and $b$, there exist $m$ functions $H^{(i)}(\mathbf{x}), \cdots H^{(m)}(\mathbf{x})$ for which it is impossible to find an algorithm using only $\mathbf{H}, \mathbf{a}, b$, providing closer points of accumulation to $\mathbf{r}$ than the algorithm (4).

Proof: Let $\mathbf{G}(\mathbf{x})=b \mathbf{x}+\mathbf{a}$,

$$
\begin{aligned}
\mathbf{H}(\mathbf{x}) & =b \mathbf{x}, \\
\mathbf{G}^{\prime}(\mathbf{x}) & =b \mathbf{x}-\mathbf{a} .
\end{aligned}
$$

$\mathbf{H}(\mathbf{x})$ is an approximation for both $\mathbf{G}(\mathbf{x})$ and $\mathbf{G}^{\prime}(\mathbf{x})$ with limits $\mathbf{r}=\frac{a}{1-b}$ and $\mathbf{r}^{\prime}=\frac{-\mathbf{a}}{1-b}$.

If any sequence $\mathrm{W}_{n}$ has a point of accumulation $\mathrm{W}$ such that

$$
|\mathbf{w}-\mathbf{r}|<\frac{|\mathbf{a}|}{1-b}
$$

then by the triangular inequality,

$$
\left|w-r^{\prime}\right|>\frac{|a|}{1-b}
$$

and the process (4) provides in this case better information.

3. Round-off Errors. For the computer, the process (1) can be written in the form

$$
y_{n+1}^{(i)}=\left[G^{(i)}\left(\mathbf{y}_{n}\right)+\xi_{n}^{(i)}\right]_{R} ;
$$

$y_{n}^{(i)}$ is an integer. [ $]_{R}$ is called a rounding procedure. $[x]_{R}$ is any integer-valued function of $x$ satisfying the inequality:

$$
\left|[x]_{R}-x\right|<1 \text {. }
$$


We consider two particular types of rounding procedures:

1) Normal rounding: $[x]_{N}=[x+0.5]$;

2) Anomalous rounding: $[x]_{A}$ : for $|x| \leqq 1,\left|[x]_{A}\right| \geqq|x|$; for $|x| \geqq 1, \quad\left|[x]_{A}\right| \leqq|x|$.

Theorem 3. Let $\mathbf{G}$ and $\xi$ satisfy equations (2) and (3). If

$$
y_{n+1}^{(i)}=\left[G^{(i)}\left(y_{n}\right)+\xi_{n}{ }^{i}\right]_{N}, \quad i=1,2 \cdots m
$$

then for any $y_{0}$, there exists $N$ such that

$$
\left|\mathbf{y}_{n}-\mathbf{r}\right| \leqq \frac{|\mathbf{a}|}{1-b}+\frac{\sqrt{m}}{2(1-b)} \quad \text { for } n>N
$$

furthermore, for given a and $b$, there exists a function $G$ and errors $\xi_{n}$ for which the bound is attained.

Now, we restrict ourselves to the particular case $m=1$; i.e., the process (1) becomes scalar. Equations (1), (2), (3), and (5) can be written as:

$$
\begin{gathered}
x_{n+1}=G\left(x_{n}\right) ; \\
|G(x)-r| \leqq b|x-r| \\
y_{n+1}=\left[G\left(y_{n}\right)+\xi_{n}\right]_{R} \\
\left|\xi_{n}\right| \leqq a
\end{gathered}
$$

Theorem 4. Let $G(x)$ and $\xi_{n}$ satisfy equations (8) and (10). If

$$
y_{n+1}=y_{n}+\left[G\left(y_{n}\right)+\xi_{n}-y_{n}\right]_{\Lambda},
$$

then for any $y_{0}$, there exists $N$ such that

$$
\left|y_{n+1}-r\right|<\frac{a}{1-b}+1 \text { for } n>N
$$

Let us compare Theorem 4 with Theorem 3 for $m=1$. In both cases, the bounds of errors have a common part which can be recognized from Theorems 1 and 2 as provided by the truncation errors. The part due to the round-off errors is independent of $b$ for the anomalous rounding; in particular, if $a=0$, the error is less than 1 and if the limit $r$ is an integer, it is reached after a finite number of steps. When the convergence is slow, i.e., $b \sim 1$, the errors can be very large for the normal rounding, even if $a=0$; however, if $b<0.5$, the normal rounding provides slightly better results than the anomalous rounding.

Remark. The condition (2) insures a first-order convergence for the process (1). If we assume higher convergence, i.e., if

$$
|\mathbf{G}(\mathbf{x})-\mathbf{r}| \leqq b|\mathbf{x}-\mathbf{r}|^{p}, \quad p>1,
$$

we get results which are quite similar, but generally not simple to formulate. Rather roughly, Theorem 4 becomes: if $y_{n}$ is computed by (11), then

$$
\left|y_{n}-r\right|<B+1 \text { for } n>N
$$

where $B$ is due to the truncation error. 


\section{Proofs.}

Lemma. Let $\mathbf{V}_{1}=\mathbf{G}\left(V_{0}\right)+\xi_{0}$ under assumptions (2) and (3);
a) If $\left|\mathbf{V}_{0}-\mathbf{r}\right| \leqq \frac{|\mathbf{a}|}{1-b}$, then $\left|\mathbf{V}_{1}-\mathbf{r}\right| \leqq \frac{|\mathbf{a}|}{1-b}$;
b) If $\left|\mathbf{V}_{0}-\mathbf{r}\right|>\frac{|\mathbf{a}|}{1-b}$, then $\left|\mathbf{V}_{1}-\mathbf{r}\right|<\left|\mathbf{V}_{0}-\mathbf{r}\right|$.

Proof. Since $\mathrm{V}_{1}=\mathbf{G}\left(\mathrm{V}_{0}\right)+\xi_{0}$ :

$$
\left|\mathbf{V}_{1}-\mathbf{r}\right| \leqq\left|\mathbf{G}\left(\mathbf{V}_{0}\right)-\mathbf{r}\right|+\left|\xi_{0}\right| \leqq b\left|\mathbf{V}_{0}-\mathbf{r}\right|+|\mathbf{a}|
$$

a) $\left|\mathbf{V}_{0}-\mathbf{r}\right| \leqq \frac{|\mathbf{a}|}{1-b} ;$ we have by (12):

$$
\left|\mathbf{V}_{1}-\mathbf{r}\right| \leqq|\mathbf{a}|\left\{\frac{b}{1-b}+1\right\}=\frac{|\mathbf{a}|}{1-b}, \text { q.e.d. }
$$

b) $\left|\mathbf{V}_{0}-\mathbf{r}\right|>\frac{|\mathbf{a}|}{1-b} ;$ we have by (12):

$$
\left|\mathbf{V}_{1}-\mathbf{r}\right| \leqq\left|\mathbf{V}_{0}-\mathbf{r}\right|-(1-b)\left|\mathbf{V}_{0}-\mathbf{r}\right|+|\mathbf{a}|<\left|\mathbf{V}_{0}-\mathbf{r}\right|-|\mathbf{a}|+
$$
$|\mathbf{a}|=\left|\mathbf{V}_{0}-\mathbf{r}\right|$, q.e.d.

Proof of Theorem 1. First case: There is $N$ such that $\left|\mathbf{V}_{N}-\mathbf{r}\right| \leqq \frac{|\mathbf{a}|}{1-b} ;$ by Lemma a, the same inequality holds for all $n>N$ and the theorem is proved.

Second case: For all $n=0,1,2, \cdots:\left|\mathbf{V}_{n}-\mathbf{r}\right|>\frac{|\mathbf{a}|}{1-b}$; by Lemma b, the positive sequence $\left|\mathbf{V}_{n}-\mathbf{r}\right|$ is monotone decreasing and converges therefore to a limit $l$.

Suppose that $l=\frac{|\mathbf{a}|}{1-b}+d$ where $d>0$; since $b<1$, there exists $\mathbf{V}_{n}$ such that $\left|\mathbf{V}_{n}-\mathbf{r}\right|<\frac{|\mathbf{a}|}{1-b}+\frac{d}{b}$; by (12):

$$
\left|\mathbf{V}_{n+1}-\mathbf{r}\right|<\frac{b}{1-b}|\mathbf{a}|+d+|\mathbf{a}|=\frac{|\mathbf{a}|}{1-b}+d=l
$$

which is a contradiction.

Proof of Theorem 3. Since $\left|[x]_{N}-x\right| \leqq 0.5$, we can write the equation (6) in the form

$$
y_{n+1}^{(i)}=G^{(i)}\left(\mathbf{y}_{n}\right)+\eta_{n}^{(i)}, \quad i=1,2, \cdots m
$$

where

$$
\left|\eta_{n}^{(i)}\right| \leqq a^{(i)}+0.5
$$

and therefore

$$
\left|\mathbf{n}_{n}\right| \leqq|\mathrm{a}|+0.5 \sqrt{\bar{m}}
$$


Replacing $\xi$ by $\mathbf{n}_{n}$ and $|\mathbf{a}|$ by $|\mathbf{a}|+0.5 \sqrt{m}$, we can apply Theorem 1: for any $\epsilon$, there exists $N$ such that

$$
\left|\mathrm{y}_{n}-\mathrm{r}\right|<\frac{|\mathrm{a}|+0.5 \sqrt{m}}{1-b}+\epsilon \text { for } n>N
$$

but since the $y_{n}^{(i)}$, s are integers, there exists a particular $\epsilon$ for which the preceding inequality implies

$$
\left|\mathbf{y}_{n}-\mathbf{r}\right| \leqq \frac{|\mathbf{a}|+0.5 \sqrt{m}}{1-b} \text { for } n>N
$$

as desired. We have still to show an example valid for every a and $b$ where the bound of error is attained. Let

$$
G^{(i)}(\mathbf{x})=b x^{(i)}-a^{(i)}-0.5
$$

and suppose that for the particular vector $y_{0}=0$ we have $\xi_{0}=a$. Then

$$
\mathbf{y}_{n}=0 \text { and }\left|\mathbf{y}_{n}-\mathbf{r}\right|=\frac{|\mathbf{a}|+\sqrt{m} \cdot 0.5}{1-b} \text { for } n \geqq 0 .
$$

Proof of Theorem 4. We use the two simple properties of the anomalous rounding procedures:

1) $x-1<[x]_{A}<x+1$;

2) If $p<x<q$ and $q-p>1$, then

$$
\begin{array}{ll}
p<p+[x-p]_{A}<q, & \text { provided that } p \text { is an integer, and } \\
p<q+[x-q]_{A}<q, & \text { provided that } q \text { is an integer. }
\end{array}
$$

Since the $y_{n}$ 's are integers, the theorem results from the three statements:

I If $\left|y_{0}-r\right| \leqq \frac{a}{1-b}$, then $\left|y_{1}-r\right|<\frac{a}{1-b}+1$;

II If $\frac{a}{1-b}<\left|y^{0}-r\right|<\frac{a}{1-b}+1$, then $\left|y_{1}-r\right|<\frac{a}{1-b}+1$;

III If $\left|y_{0}-r\right| \geqq \frac{a}{1-b}+1$, then $\left|y_{1}-r\right|<\left|y_{0}-r\right|$.

Statement I: By Lemma $a$ :

$$
r-\frac{a}{1-b} \leqq y_{0}+G\left(y_{0}\right)+\xi_{0}-y_{0} \leqq r+\frac{a}{1-b} ;
$$

By property 1:

$$
\begin{gathered}
r-\frac{a}{1-b}-1<y_{0}+\left[G\left(y_{0}\right)+\xi_{0}-y_{0}\right]_{A}<r+\frac{a}{1-b}+1 ; \text { i.e. } \\
\left|y_{1}-r\right|<r+\frac{a}{1-b}+1, \text { q.e.d. }
\end{gathered}
$$

Statement II: We suppose $r+\frac{a}{1-b}<y_{0}<r+\frac{a}{1-b}+1$ (the proof is 
analogous, when

$$
\begin{gathered}
\left.r-\frac{a}{1-b}-1<y_{0}<r-\frac{a}{1-b}\right) ; \text { by Lemma } b: \\
p \equiv r-\frac{a}{1-b}-1<y_{0}+G\left(y_{0}\right)+\xi_{0}-y_{0}<y_{0} \equiv q ; \text { since } \\
y_{0}>r, \quad q-p>1 \text { and we apply property } 2: \\
r-\frac{a}{1-b}-1<y_{0}+\left[G\left(y_{0}+\xi_{0}-y_{0}\right)\right]_{A}<y_{0}<r+\frac{a}{1-b}+1 ; \text { i.e., } \\
\quad\left|y_{1}-r\right|<r+\frac{a}{1-b}+1 \text {, q.e.d. }
\end{gathered}
$$

Statement $I I I$ : We suppose $y_{0} \geqq r+\frac{1}{1-b}+1$ (the proof is analogous when

$$
\begin{gathered}
\left.y_{0} \leqq r-\frac{a}{1-b}-1\right) ; \quad \text { by Lemma } b: \\
p \equiv 2 r-y_{0}<y_{0}+G\left(y_{0}\right)+\xi_{0}-y_{0}<y_{0} \equiv q ;
\end{gathered}
$$

by property 2 , since $q-p>1$ :

$$
\begin{gathered}
2 r-y_{0}<y_{0}+\left[G\left(y_{0}\right)+\xi_{0}-y_{0}\right]_{A}<y_{0} ; \text { i.e., } \\
\left|y_{1}-r\right|<\left|y_{0}-r\right|, \quad \text { q.e.d. }
\end{gathered}
$$

\section{APPENDIX I: Iterative Processes with a Floating-Point Computer*}

Let $r$ be a real number and $G(x)$ be a function such that

$$
|x+G(x)-r| \leqq b|x-r| \text { with } 0 \leqq b<1 \text { for any } x
$$

then the sequence

$$
x_{n+1}=x_{n}+G\left(x_{n}\right)
$$

converges at least linearly to $r$ for any $x_{0}$.

Suppose we want to realize (2) on a binary floating-point computer, i.e., the numbers are of the form $\alpha \cdot 2^{\beta}$, where $\alpha$ is an exact binary fraction and $\beta$ is an integer.

A number will be called normalized if 1) $0.5 \leqq|\alpha|<1$;2) $\alpha$ is an exact binary fraction representable by $N$ bits and the sign; 3$) \beta \geqq-p(N$ and $p$ are fixed numbers); furthermore there exists a real zero, representable for example by $\alpha=0$, $\beta=-p$; for greater simplicity, this zero will also be included in the class of normalized numbers.

We assume that in the realization of (2) on the computer, both $x_{n}$ and $G\left(x_{n}\right)$ are represented by normalized numbers; of course $G(x)$ cannot be computed exactly in general; so we assume that value effectively computed, $\bar{G}(x)$, satisfies the relation:

$$
\bar{G}(x)=(1+\eta) G(x)+\zeta ; \quad|\eta| \leqq d,|\zeta| \leqq a ;
$$

* A detailed discussion of the results of this appendix will be found in reference [4]. 
where $\eta$ and $\zeta$ are functions of $x$, but $d$ and $a$ are fixed numbers.

The effective process is given by the operation

$$
Y_{n+1}=\left[Y_{n}+\bar{G}\left(Y_{n}\right)\right]_{R}
$$

where $Y_{n}$ and $Y_{n+1}$ are normalized numbers; since $Y_{n}+\bar{G}\left(Y_{n}\right)$ cannot be generally represented by a normalized number, it must be rounded as indicated by []$_{R}$.

We concentrate our attention on the rounding procedure in (4) and consider two types of rounding procedures:

1) Normal rounding. $Y_{n+1}=\left[Y_{n}+\bar{G}\left(Y_{n}\right)\right]_{N} ; Y_{n+1}$ is a normalized number such that

$$
\left|Y_{n+1}-\left(Y_{n}+\bar{G}\left(Y_{n}\right)\right)\right|=\text { minimum; }
$$

when two different normalized numbers satisfy the above relation, either of them can be chosen as $Y_{n+1}$.

2) Anomalous rounding. $Y_{n+1}=\left[Y_{n}+\bar{G}\left(Y_{n}\right)\right]_{A}$; if $\bar{G}\left(Y_{n}\right) \geqq 0$ let

$Z$ be the smallest normalized number such that $Z \geqq Y_{n}+\bar{G}\left(Y_{n}\right)$,

$W$ be the greatest normalized number such that $W \leqq Y_{n}+\bar{G}\left(Y_{n}\right)$;

if $\bar{G}\left(Y_{n}\right) \leqq 0$ let

$Z$ be the greatest normalized number such that $Z \leqq Y_{n}+\bar{G}\left(Y_{n}\right)$,

$W$ be the smallest normalized number such that $W \geqq Y_{n}+\bar{G}\left(Y_{n}\right)$; then

$$
\begin{array}{lll}
{\left[Y_{n}+\bar{G}\left(Y_{n}\right)\right]_{A}=W} & \text { if } & W \neq Y_{n} \\
{\left[Y_{n}+\bar{G}\left(Y_{n}\right)\right]_{A}=Z} & \text { if } & W=Y_{n} .
\end{array}
$$

Theorem. a) For any $Y_{0}$, by using normal rounding in (4), there exists a finite number $M$ such that

$$
\left|Y_{n}-r\right| \leqq B_{N} \equiv \frac{2^{-N}|r|+a\left(1+2^{-N}\right)}{2+2^{-N}-(1+d)(1+b)\left(1+2^{-N}\right)} \quad \text { for } \quad n>M .
$$

b) For any $Y_{0}$, by using anomalous rounding in (4), there exists a finite number $M$ such that

$$
\left|Y_{n}-r\right|<B_{\Lambda} \equiv|r| 2^{-N+1}+2^{-p-1}+\frac{a\left(1+2^{-N+1}\right)}{2-(1+d)(1+b)} \text { for } n>M \text {. }
$$

If $B_{N}$ or $B_{A}$ is negative, it must be replaced by $+\infty$.

In order to compare these results, first suppose $a=0$. Then $B_{A}$ is independent of $b$ and $d$ and furthermore remains very small; in case of slow convergence, i.e., when $b \cong 1, B_{N}$ can become very large. The increase of magnitude of the bounds when $a>0$ is almost the same for $B_{A}$ and $B_{N}$ for reasonable cases, so that the anomalous rounding can be considered safer than the normal rounding.

Remarks. 1) The relations of normal and anomalous rounding procedures are very similar in fixed-point and in floating-point arithmetics;

2 ) The bounds $B_{A}$ and $B_{N}$ are reached only in trivial cases; however, examples show that they remain realistic in every case. 


\section{APPENDIX II: Round-off Errors in Aitken's $\delta^{2}$ Process*}

Let $G(x)$ be a real continuous function of the real variable $x$ such that the sequence $x_{n}$ defined by

$$
x_{n+1}=G\left(x_{n}\right)
$$

converges to the limit $x=r$.

By Aitken's $\delta^{2}$ process, we define another sequence:

$$
\left\{\begin{array}{l}
V_{3 n+1}=G\left(V_{3 n}\right) \\
V_{3 n+2}=G\left(V_{3 n+1}\right) \\
V_{3 n+2}=\frac{V_{3 n} V_{3 n+2}-V_{3 n+1}^{2}}{V_{3 n}+V_{3 n+2}-2 V_{3 n+1}}
\end{array}\right.
$$

Let us suppose we want to realize process (2) on a fixed-point computer with the following conditions: a) We use only one "word" for representing the $V_{i}$ 's; we may consider the content of the word as an integer; b) We may use higher precision for computing $G\left(V_{i}\right)$.

We cannot expect to compute $G\left(V_{i}\right)$ without error; furthermore, if we are using higher precision, the result must be rounded to an integer.

Definition. A rounding procedure denoted by $[x]_{R}$ is any integer-valued function of the real variable $x$ satisfying the inequality:

$$
\left|[x]_{R}-x\right|<1
$$

We shall use the following particular rounding procedures:

1) $[x]^{\nearrow}$ : rounding away from zero; it is defined by the inequality

$$
\left|[x]^{\nearrow}\right| \geqq|x| \text {; }
$$

2) $[x]^{\swarrow}$ : rounding toward zero; it is defined by the inequality

$$
\left|[x]^{\swarrow}\right| \leqq|x| \text {. }
$$

Example. Let $G(x)=7 / 8 x$ and $V_{0}=8$; by (2), we have

$$
\begin{aligned}
& V_{1}=7 \\
& V_{2}=6,125 \\
& V_{3}=0 .
\end{aligned}
$$

If we want to represent the $V_{i}$ 's only by integers and if we use the normal rounding procedure, we shall find:

$$
\begin{aligned}
& \bar{V}_{1}=7 \\
& \bar{V}_{2}=6 \\
& \bar{V}_{3}=\infty .
\end{aligned}
$$

\footnotetext{
* For the proof see reference [3], part II.
} 
This situation can be improved by using the following integer process:

$$
\left\{\begin{array}{l}
W_{3 n+1}=W_{3 n}+\left[G\left(W_{3 n}\right)+\xi_{3 n}-W_{3 n}\right]^{\nearrow} \\
W_{3 n+2}=W_{3 n}+\left[G\left(W_{3 n+1}\right)+\xi_{3 n+1}-W_{3 n}\right]^{\swarrow} \\
W_{3 n+3}=W_{3 n}+\left[\frac{\left(W_{3 n}-W_{3 n+1}\right)^{2}}{2 W_{3 n+1}-W_{3 n}-W_{3 n+2}}\right] .
\end{array}\right.
$$

$\xi_{3 n}$ and $\xi_{3 n+1}$ are the errors of computation of $G\left(W_{3 n}\right)$ and $G\left(W_{3 n+1}\right)$; since the numerator and the denominator are integers, it is possible with the help of the remainder to compute $W_{3 n+3}$ without any error; if the numerator and the denominator are simultaneously equal to zero, then $W_{3 n}=W_{3 n+1}=W_{3 n+2}$ and we set $W_{3 n+3}=W_{3 n}$.

Theorem 1. We suppose there exist numbers $0 \leqq b<1, \quad 0 \leqq c<1, \quad \delta \geqq 0$ such that:

$$
\left|x_{1}-r\right| \leqq b\left|x_{0}-r\right|
$$

for any $x_{0}$ and $x_{1}$ satisfying the relation (1);

$$
\left|V_{3}-r\right| \leqq c\left|V_{0}-r\right|
$$

for any $V_{0}$ and $V_{3}$ satisfying the relations (2);

$$
|G(x)-G(y)| \leqq \delta|x-y|
$$

for any $x$ and $y$;

4) the errors $\xi_{3 n}$ and $\xi_{3 n+1}$ in (3) satisfy the inequality

$$
\left|\xi_{j}\right| \leqq a \leqq d=\frac{1}{4} \frac{(1-b)^{2}(1-c)}{(1+c)(1+\delta)} ;
$$

then, for any $W_{0}$ there exists a finite number $N$ such that

$$
\left|W_{3 n}-r\right|<1+\frac{a}{1-b} \text { for } n>N .
$$

THeOREm 2. We make the assumptions:

1) The convergence of process (1) is alternating, i.e., for any $x$

$$
\begin{array}{lll}
0 \leqq r-G(x)<x-r & \text { if } & x-r>\mathrm{C}, \\
0 \leqq G(x)-r<r-x & \text { if } & x-r<0 . \\
G(x)=r & \text { if } & x=r ;
\end{array}
$$

2) The errors $\xi_{3 n}$ and $\xi_{3 n+1}$ in (3) satisfy the inequality

$$
\left|\xi_{j}\right| \leqq a \leqq \frac{1}{3},
$$

where $a$ is a fixed number; then, for any $W_{0}$ there exists a finite number $N$ such that

$$
\left.\mid W_{3 n}-r\right] \leqq 1+a \text { for } n>N \text {. }
$$

Remark. Assumption (1) of Theorem 2 is sufficient for providing the conver- 
gence of the $V_{n}$ 's satisfying the equations (2) for any $V_{0}$. It is easy to prove the inequality:

$$
\left|V_{3 n}-r\right|<\frac{\left|V_{0}-r\right|}{3^{n}}
$$

University of Illinois

Urbana, Illinois

1. A. S. Householder, Principles of Numerical Analysis. McGraw-Hill, 1953.

2. ARNOLD NORDSIECK, "On numerical integration of ordinary differential equations," Math. Comp., v. 16, January, 1962.

3. JEAN Descloux, Remarks on the Round-Off Errors in Iterative Processes for Fixed-Point Computers. University of Illinois, Digital Computer Laboratory, Urbana, Illinois, Report No. 116, May, 1962.

4. JEAN Descloux, Remarks on Errors in First-Order Iterative Processes with FloatingPoint Computers. University of Illinois, Digital Computer Laboratory, Urbana, Illinois, Report No. 113, March, 1962. 